\title{
EXPECTED EMITTANCE GROWTH AND BEAM TAIL REPOPULATION FROM ERRORS AT INJECTION INTO THE LHC
}

\author{
B. Goddard, H. Burkhardt, V. Kain, T. Risselada, CERN, Geneva, Switzerland
}

\begin{abstract}
The preservation of the transverse emittance of the proton beam at injection into the LHC is crucial for luminosity performance. The population of the beam tails is also important for beam losses and collimation. The transfer and injection process is particularly critical in this respect, and several effects can contribute to the expected emittance increase and tail repopulation, like optical and geometrical mismatch, injection offsets and coupling, etc. The various effects are described, together with the tolerance limits on the parameters, and the expected contributions evaluated analytically where possible. The emittance growth and tail distributions are also simulated numerically using realistic errors. The implications for the tolerances on the matching of the transfer lines are discussed.
\end{abstract}

\section{INTRODUCTION}

The total emittance budget between the SPS extraction and LHC colliding beams is only $\Delta \epsilon / \epsilon_{0} \leq 1.07$ [1], which places stringent requirements on the various mismatch factors for transfer and injection. For the effects identified below, each contribution should be below a few \%, and the total below 5

The details of the particle distributions are also important, since large tail populations can cause transient losses at injection which risk quenching superconducting magnets. The beams will be scraped to about $3.5 \sigma$ in the SPS, but some mismatch effects (for example the geometrical tilt) can lead to significant tail repopulation at large amplitudes, although the effect on the emittance may remain small.

\section{MISMATCH EFFECTS}

\section{Steering error}

An injection angle or position error leads to to an emittance increase [2] of:

$$
\frac{\epsilon}{\epsilon_{0}}=1+\frac{1}{2} \frac{\Delta x^{2}+\left(\beta \Delta x^{\prime}+\alpha \Delta x\right)^{2}}{\beta \epsilon_{0}}=1+\frac{1}{2} \Delta e^{2}
$$

In the LHC the injection errors are damped, and the emittance increase after damping is given by:

$$
\frac{\epsilon}{\epsilon_{0}}=1+\frac{1}{2} \Delta e^{2}\left(\frac{1}{1+\tau_{D C} / \tau_{d}}\right)^{2}
$$

where $\tau_{D C}$ and $\tau_{d}$ are the $68 \mathrm{~ms}$ filamentation time and 5 ms damping time, respectively [3]. The $1.5 \sigma$ maximum injection error gives an emittance increase of $0.5 \%$.

\section{Betatron mismatch}

The $\alpha$ and $\beta$ of the injected beam may not match those of the LHC, due to errors in the SPS, transfer line or LHC. For a mismatch factor $\lambda$ the emittance increase [4] is:

$$
\frac{\epsilon}{\epsilon_{0}}=\frac{1}{2}\left(\lambda^{2}+\lambda^{-2}\right)=\frac{1}{2}\left(\beta_{1} \gamma_{2}+\beta_{2} \gamma_{1}-2 \alpha_{1} \alpha_{2}\right)
$$

where subscripts 1 and 2 refer to the matched (lattice) and mismatched (beam) ellipses, respectively. The TI 8 tests in 2004 [5] showed that $\lambda$ could be expected to be corrected to about 1.10. The total mismatch is estimated at 1.15 , which includes a $5 \%$ margin for drifts of the LHC optics during the injection process. The emittance increase from this source is then $3.9 \%$.

\section{Dispersion mismatch}

The dispersion may be mismatched at the injection point. For dispersion mismatch factors $\delta D, \delta D^{\prime}$, a relative momentum spread $\delta p / p$ gives an emittance increase [2] of:

$$
\frac{\epsilon}{\epsilon_{0}}=1+\frac{1}{2} \frac{\Delta D^{2}+\left(\beta \Delta D^{\prime}+\alpha \Delta D\right)^{2}}{\beta \epsilon_{0}}\left(\frac{\delta p}{p}\right)^{2}
$$

The expected dispersion mismatch is of the order of $0.2 \mathrm{~m}$ and $0.002 \mathrm{rad}$, for $\Delta D$ and $\Delta D^{\prime}$ respectively, which include contributions of the order of $0.05 \mathrm{~m}$ from imperfect matching to the separation and crossing bumps at the injection points, together with about $0.15 \mathrm{~m}$ of spurious dispersion in the LHC ring. With the nominal energy spread of $5 \times 10^{-4}$, the emittance increase is $2.4 \%$.

\section{Energy error}

With dispersion $\mathrm{D}$ at the injection point and an energy error of $\Delta p / p$, the emittance increase is:

$$
\frac{\epsilon}{\epsilon_{0}}=1+\frac{1}{2} \frac{D^{2}}{\beta \epsilon_{0}}\left(\frac{\Delta p}{p}\right)^{2}
$$

For the nominal dispersion of about $0.1 \mathrm{~m}$, an energy error of $5 \times 10^{-4}$ gives an emittance increase of $0.2 \%$. 


\section{Geometrical (tilt) mismatch}

There is a geometrical mismatch in the transfer lines due to the uncorrected rotation between the beam $\mathrm{XZ}$ plane and that of the LHC [6]. If $\theta$ is the angle between these planes, the emittance increase in the horizontal plane for $\epsilon_{0 x}=\epsilon_{0 y}$ is:

$$
\frac{\epsilon_{x}}{\epsilon_{0 x}}=1+\frac{1}{2}\left(\beta_{x} \gamma_{y}+\beta_{y} \gamma_{x}-2 \alpha_{x} \alpha_{y}-2\right) \sin ^{2} \theta
$$

with an equivalent expression for the vertical plane. For the 52 mrad angle between TI 8 and the LHC, the emittance increase is $1.3 \%$. For TI 2, with a $20 \mathrm{mrad}$ angle, the expected emittance increase is $0.3 \%$.

\section{Coupling}

For a coupling factor $\kappa=\bar{y} / \bar{x}$ (the amplitude of an oscillation launched in the $\mathrm{x}$ plane coupled into the $\mathrm{y}$ plane), the emittance increase is given simply by:

$$
\frac{\epsilon_{x}}{\epsilon_{0 x}}=1+\frac{1}{2} \kappa^{2}
$$

For the LHC transfer lines $\kappa$ should be zero, and was measured in 2004 in TI 8 at 3\% [5]. The resulting emittance increase is $0.05 \%$.

\section{Scattering from beam instrumentation screens}

The rms Multiple Coulomb scattering angle $\theta_{s}$ for $450 \mathrm{GeV}$ protons is given by the screen thickness $L$ and material radiation length $L_{\text {rad }}(\gg L)$ :

$$
\theta_{s} \approx 3.13 \times 10^{-5} \sqrt{L / L_{\text {rad }}}
$$

At a screen location with beta function $\beta$, the emittance increase [4] is given by:

$$
\frac{\epsilon}{\epsilon_{0}}=1+\frac{1}{2} \frac{\beta}{\epsilon_{0}} \theta_{s}^{2}
$$

The LHC transfer line screens are made of $12 \mu m$ thick titanium, with $L_{r a d}=0.036 \mathrm{~m}$. The $\beta$ functions at the screens are in the range $60-220 \mathrm{~m}$. The emittance increase from one screen is $0.1-0.55 \%$ per plane.

\section{Summary of expected contributions}

The various contributions are given in Table 1. Assuming that these add quadratically, the expected emittance increase is $4.8 \%$, compared to the $7 \%$ budget. It is assumed that no screens are left in the line during LHC filling; however, their individual contributions are small, of the order of $0.5 \%$, and so the presence of several screens would be acceptable (10 such screens left in the line would increase the emittance by about $1.5 \%$ ).
Table 1: Estimated contributions to the emittance increase for transfer and injection into the LHC (TI 8 values).

\begin{tabular}{|l|c|c|c|c|}
\hline Error & Parameter & Unit & Value & $\Delta \epsilon / \epsilon_{0}$ \\
\hline $\begin{array}{l}\text { Steering } \\
\text { (damped) }\end{array}$ & $\begin{array}{c}\Delta e \\
\tau_{D C} / \tau_{d}\end{array}$ & $\sigma$ & 1.5 & \\
\hline Betatron & $\lambda$ & & 14 & 1.005 \\
\hline Dispersion & $\Delta D$ & $\mathrm{~m}$ & 0.20 & 1.039 \\
& $\Delta D^{\prime}$ & $\mathrm{rad}$ & 0.002 & 1.024 \\
\hline Energy & $\Delta p / p$ & & $5 \cdot 10^{-4}$ & 1.002 \\
\hline Tilt & $\theta$ & $\mathrm{rad}$ & 0.052 & 1.013 \\
\hline Coupling & $\kappa$ & & 0.03 & 1.001 \\
\hline \hline Total & & & & $\mathbf{1 . 0 4 8}$ \\
\hline
\end{tabular}

\section{MONTE-CARLO SIMULATIONS}

To obtain a more realistic picture of how the expected effects combine, the emittance increase and tail repopulation were estimated numerically by a Monte-Carlo simulation. All contributing effects were incorporated with systematic and random components, including trajectory correction, power supply ripples, extraction and injection kicker waveforms, energy error and spread, optical (betatron and dispersion) mismatch, tilt mismatch and magnet and BPM misalignments. The study was made for TI 8 .

\section{Emittance increase}

For 1000 seeds, 10,000 particles were produced with a realistic scraped initial distribution [7], and the optics perturbations calculated for the line with all the errors. The transformed particle distributions were produced at the injection point, and the new phase space amplitude distributions and emittance increases calculated. The initial and final phase-space distribution for particles in the horizontal plane for one seed can be seen in Fig. 1 (here using a uniform initial distribution for illustration). The tilt mismatch effect is just discernible.

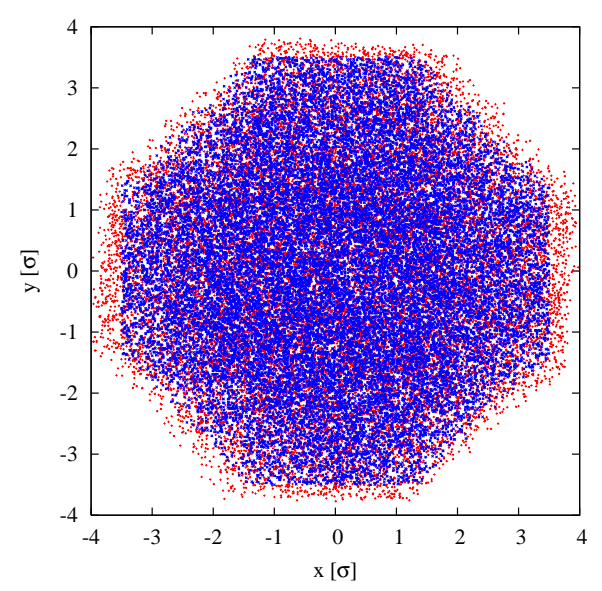

Figure 1: Initial (blue) and transformed (red) particle phase-space distribution.

The results for the relative emittance increases with scraped Gaussian beams are shown in Fig. 2. The max- 
imum values observed are 7-10\%, larger than the corresponding analytical estimate of $4.8 \%$ above. The average (mean) increase is $2.8 \%$ for the $\mathrm{H}$ plane and $2.7 \%$ for $\mathrm{V}$; the $95 \%$ confidence limits are at $5.9 \%$ and $4.4 \%$ for $\mathrm{H} / \mathrm{V}$, respectively.
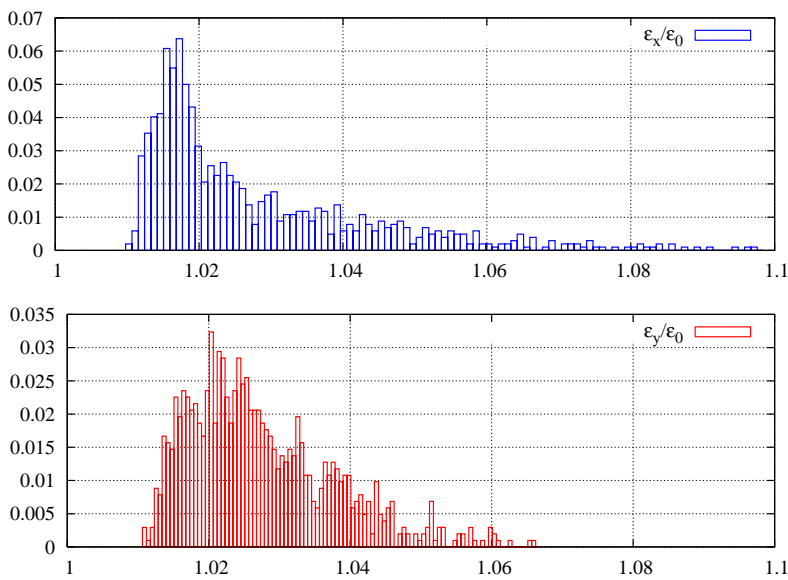

Figure 2: Distribution of expected emittance increases for transfer into LHC ring 2, via TI 8.

\section{Particle distributions and tail repopulation}

To estimate the tail repopulation with all mismatch effects, transformed distributions of 500,000 particles were compared with the initial distributions. A typical result is shown in Fig. 3, where it can be seen that the tails of the distribution are repopulated to beyond $4 \sigma$.

160 seeds were considered. The edge of the final distribution was estimated by measuring the particle amplitude at the $10^{-5}$ level. The distribution of the resulting tail repopulation for the 160 seeds is shown in Fig. 4. The average (mean) repopulation is to $3.86 \sigma$ in $\mathrm{H}$ and $3.98 \sigma$ in $\mathrm{V}$, with $95 \%$ confidence limits at $4.03 \sigma$ and $4.13 \sigma$ for $\mathrm{H} / \mathrm{V}$, respectively. The repopulated distribution appears Gaussian to within about $0.2 \sigma$ of these values.
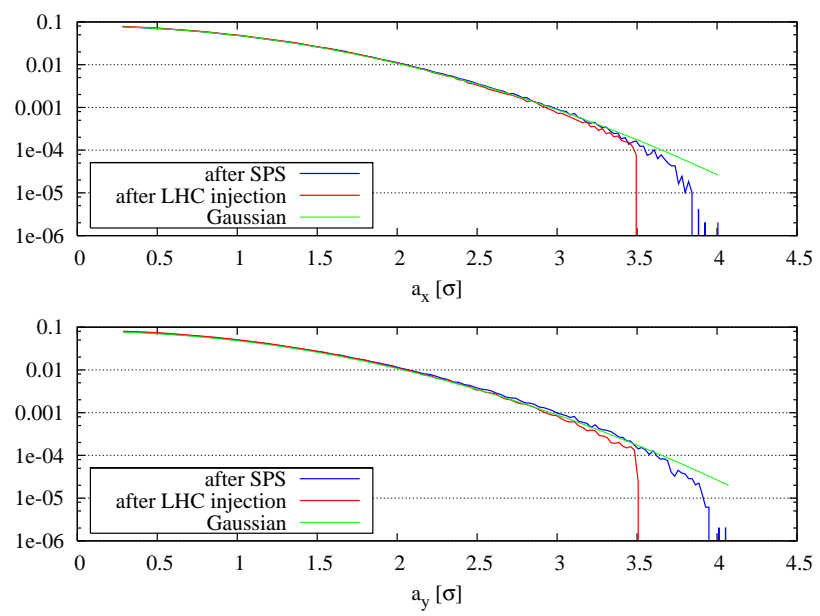

Figure 3: Initial (red) and repopulated (blue) tails, which stay Gaussian out to about $3.8-4 \sigma$.
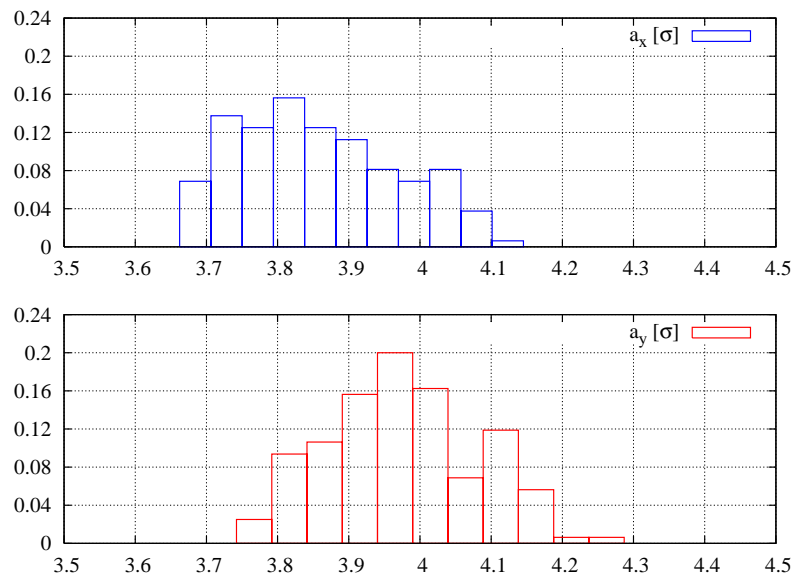

Figure 4: Distribution of tail repopulation for 160 seeds.

\section{DISCUSSION}

The maximum emittance increases observed from the full Monte-Carlo simulations confirm the analytical estimates. The results indicate an average emittance increase of $3 \%$, with a maximum of $7-10 \%$, and show that the transfer and injection process are expected to meet the design specification of $5 \%$ emittance increase at a $95 \%$ confidence limit. The most critical effects have been identified; optical mismatch (both betatron and dispersion) and tilt mismatch. The latter is a design feature, with currently no correction planned. The betatron mismatch $\lambda$ must be kept to $\leq 1.15$, including static and dynamic effects on the injection plateau. The dispersion mismatch must be kept to $\sim 0.2 \mathrm{~m}$, including changes of bump polarity and spurious dispersion in the LHC. The presence of a few screens will not increase the emittance significantly.

The repopulation of the tails was also quantified, with simulations showing an average repopulation to $3.9 \sigma$, which comes mainly from the tilt mismatch. This may be an issue for clean injection; if it proves problematic the scraping depth in the SPS could be made deeper, or in the long term, a correction studied for the tilt mismatch.

\section{REFERENCES}

[1] M. Benedikt et al. (eds), LHC Design Report, Volume III, CERN, 2004.

[2] L. Vos, Mismatch, Damping and Emittance Growth, IXth CERN Chamonix Workshop, CERN-OPEN-99-074, 1999.

[3] D. Boussard, W. Höfle, T. Linnecar, The LHC Transverse Damper (ADT) Performance Specification, CERN SL-Note99-055 HRF, 1999.

[4] S. Turner (ed.), Proceedings 5th CERN Accelerator School, CERN 94-01, 1994.

[5] J. Wenninger et al., Optics Studies of the LHC Beam Transfer Line TI 8, these proceedings.

[6] B. Goddard et al., Geometrical alignment and associated beam optics issues of transfer lines with horizontal and vertical deflection, CERN LHC Project Report 719, 2004.

[7] H. Burkhardt, R. Schmidt, Intensity and Luminosity after beam Scraping, CERN AB-2004-032-ABP, 2004. 